\title{
Knowledge brokering in public health: A tale of two studies
}

Robyn Traynor, M.Sc. ${ }^{1}$, Kara DeCorby, M.Sc. ${ }^{1}$, Maureen Dobbins, R.N., Ph.D. ${ }^{1, \S}$

${ }^{1}$ Faculty of Health Sciences, McMaster University, 1200 Main Street West, Hamilton, Canada

${ }^{\S}$ Corresponding author: Maureen Dobbins (MD)

E-mail address:

RT: $\quad$ rtraynor@healthevidence.org

KD: kdecorby@healthevidence.org

MD: dobbinsm@mcmaster.ca 


\begin{abstract}
Objectives: A Knowledge Broker is one approach for facilitating the integration of evidenceinformed decision making in public health practice. In this paper, the findings from two studies investigating a Knowledge Broker intervention as a means of enhancing capacity for evidenceinformed decision making are presented. Contextual factors that facilitate this strategy are also identified.
\end{abstract}

Study Design: This paper describes work done through a single mixed-methods study (randomized controlled trial with a qualitative component) and a case study.

Methods: The Health Evidence team conducted two studies examining Knowledge Broker impact in Canadian public health departments. The effectiveness of knowledge translation strategies of varying intensities for promoting the use of research evidence in decisions related to child obesity prevention were explored via a randomized controlled trial with a fundamental descriptive component (2003 - 2007). In a case study (2010-2013), we partnered with three health departments to develop and implement tailored strategies targeted at the organization. Knowledge Brokers worked with designated staff in these studies via one-on-one consultations, small group meetings, and/or workshops and presentations. The Knowledge Broker role was assessed by analysing data from close-ended surveys, interviews, organizational documents, and reflective journals. 
Results: In this paper, the authors focus on findings from the qualitative analysis of implementing the Knowledge Broker role in both studies and explore several contextual factors that impacted study outcomes. Knowledge Brokers were shown to enhance individual capacity by improving knowledge and skill in searching for, critically appraising, and applying research evidence to practice-based issues. Organizational capacity was also enhanced with strong management support and policies. Effective Knowledge Broker attributes included both expertise in research methodology and public health, as well as intangible traits such as approachability and patience. Finally, optimal positioning and ways of working were identified, including the importance of in-person meetings and neutrality of the Knowledge Broker.

Conclusions: Knowledge brokering is a potentially promising knowledge translation strategy for public health, though additional feasibility and cost-effectiveness data are still needed. The research presented here further highlights the importance of context and adopting a tailored approach to implement a Knowledge Broker strategy.

\section{Keywords}

Knowledge broker; knowledge translation; evidence-informed decision making; public health 


\section{Introduction}

In public health and in healthcare more broadly, there is pressure to ensure that interventions are informed by research evidence, in an aim to achieve effectiveness and also economy. ${ }^{1}$ Research evidence includes primary studies, systematic reviews, and meta-analyses. ${ }^{2}$ Research evidence represents one input in the process of evidence-informed decision making (EIDM). Other inputs into the process include public health expertise, the local and political context, and available resources. ${ }^{3}$ The field of knowledge translation (KT) promotes the use of research evidence in health care and has generally advocated for more "active" KT strategies to promote the uptake of information (e.g. change agency), rather than "passive" strategies (e.g. publications, website postings, mailouts to target audiences), while addressing barriers and facilitators. ${ }^{4-9}$ At the same time, there has been an increasing demand for meaningful decision maker involvement in research ${ }^{10,11}$ and greater emphasis on the personal factors related to KT, including trust and relationship building in this collaborative work. ${ }^{12}$

The role of a Knowledge Broker (KB) has been described as one that "links researchers and decision makers, facilitating their interaction so that they are better able to understand each other's goals and professional culture, influence each other's work, forge new partnerships and use research-based evidence. Brokering is ultimately about supporting EIDM in the organization, management and delivery of health services" (page 2). ${ }^{13}$ A key feature for KBs is this connector function, both between and among stakeholders, including researchers, practitioners, and policy makers. ${ }^{14-21}$

As an agent for change, a KB's role is based on the premise that interpersonal contact improves the likeliness of behaviour change ${ }^{22}$, making linkage and exchange a central part of the 
role. ${ }^{16: 20} \mathrm{~A} \mathrm{~KB}$ should also aim to improve skills for accessing and using research evidence ${ }^{23,24}$, with the goal of increasing readiness for uptake within the culture in which their clients work. ${ }^{16}$

The literature identifies key features and necessary skills for brokering and considerations for implementing and supporting KBs in their KT efforts. A KB must be skilled in research interpretation ${ }^{25-27}$ and possess strong network ties that assist in knowledge exchange ${ }^{26,28-30}$, and should also be able to forge new connections across domains. ${ }^{31-34}$ The strength of a KB's networks assists in information sharing and access to relevant, current knowledge. A key feature of brokering is the ability to recombine existing evidence to form new solutions ${ }^{32,35}$, capturing and sharing tacit knowledge across domains ${ }^{30,36}$, often by developing, operating, or acting as a knowledge repository. $25,27,29,33,34,36$

In addition to required skills, KBs need to be credible ${ }^{16,20}$ and have the ability to gain the trust $^{22}$ and respect ${ }^{22,37}$ of their clients. A recently released realist synthesis also indicates that a KB must be accountable, a role model, accessible, organized, and an expert. ${ }^{37}$ Being culturally compatible (i.e. having a perceived connection with the target group), reflective, and having a positive attitude were also identified as key traits. ${ }^{37}$

Brokering has been implemented widely in private industry ${ }^{26,26,28-33,35,36,38,39}$ and more recently in healthcare policy and practice. ${ }^{20,25,27,34,40}$ The role has been implemented using various KT models in different settings, yet despite variations in the application of the role across contexts, there are many commonalities, with the key feature being the linking of knowledge producers and users to facilitate interaction and promote uptake..$^{20,26,41}$

Although most evidence related to brokering is anecdotal ${ }^{42}$, projects to date suggest that brokering may be an effective way to improve the quality and usefulness of evidence employed in healthcare decision making. ${ }^{25,41}$ Local context has demonstrated a great effect on diffusion pattern 
and rate of uptake $e^{43}$, making it an important consideration in brokering efforts. Regardless of purpose or audience, a KB should translate information clearly and in a way that is transparent to users. $^{44}$

Despite the supposed potential and increasing recognition and implementation of the KB role within healthcare organizations, its effectiveness has yet to be fully established. Many questions remain about whether KBs are an effective KT strategy and how the role can be implemented to optimize impact. This paper explores the implementation of the KB role in public health, as a means of facilitating the consistent inclusion of research evidence in program decision making by public health practitioners. The results of qualitative analysis from two studies - a mixedmethods randomized controlled trial $(\mathrm{RCT})^{45}$ and a case study - will be discussed. The two studies reported here represent research data that support the anecdotal evidence that KBs are a promising strategy for EIDM. Quantitative findings that report the impact of the KB intervention will be briefly presented, although the focus of this paper is a description of the implementation of the KB intervention and reflection on the impact of organizational context on the KB's activities.

\section{Methods}

This paper describes a "health department" as an organizational unit which, to varying degrees, makes its own decisions regarding programs, policies and resource allocation. Each province and territory in Canada has its own unique public health structure and functions; as such, the organizational structure of each health department that participated in these studies differed. 
The results of two successive studies are reported here, with knowledge obtained in the earlier study informing the design, data collection and intervention in the succeeding study. Data were analyzed separately with themes developed separately for each study, based primarily on the KB reflective journal and qualitative interviews, and synthesized following completion of both studies. While we have not conducted a formalized cross-study analysis between the RCT and case study, we compared the emerging themes that spanned both studies. Each theme presented here represents a combination of evidence from both studies, based on frequency, emphasis of themes observed across various data sources within each study, and the extent to which themes were reported by most (a high proportion) participants in each study. This method did not employ a vote-counting system to identify themes.

A description of the $\mathrm{KB}$ role in the RCT is provided below. The KBs followed the same philosophy and guiding KT framework throughout both studies, although the role was modified for the case study based on lessons learned from previous work. More detailed accounts of activities and day-to-day work of the KB intervention in both studies have been described elsewhere. ${ }^{45,46}$ (Dobbins et al. unpublished work, Greco et al. submitted) The principles of fundamental qualitative data collection and analysis described by Miles and Huberman ${ }^{47}$ and Patton $^{48}$ guided both studies, while the principles of fundamental case study design described by $\mathrm{Yin}^{49}$ further informed the second study.

\section{Knowledge Broker RCT (2003-2007)}

Health departments were randomly allocated to one of three progressively more active KT interventions: 1) access to review-level evidence in an online registry at healthevidence.org; 2) tailored email messages with embedded links to evidence summaries of high quality 
systematic reviews plus registry access; and 3) KB services (i.e. the "KB intervention"), plus registry access and tailored messages. More specifically, KB services included: initial and ongoing needs assessments; scanning the horizon (i.e. remaining up-to-date on new evidence and resources in relevant content areas); knowledge management (e.g. managing published and unpublished evidence, internal evaluation information, etc.); internal network development, maintenance and facilitation; facilitation of individual capacity development in EIDM; and facilitation of and support for organizational change. ${ }^{23,45,46}$

Thirty health departments were randomized to receive the KB intervention. Impact of the $\mathrm{KB}$ intervention was measured quantitatively as the uptake of research evidence into policy and practice. In addition, qualitative data was collected via 12 interviews with selected participants to further describe the $\mathrm{KB}$ role during the one-year intervention and assess participant perception of the impact of the KB intervention on them and their organization, with respect to knowledge, attitude and behaviours related to EIDM. The interviews were recorded and transcribed verbatim, with light editing (i.e. fillers ("ums", "ahs"), ambient sounds, and non-verbal communication (pauses) were omitted). All identifying information was removed from final transcripts to ensure participant anonymity and confidentiality. Interview transcripts and journal entries were analyzed by two reviewers using NVivo 7.0. Initially, a selection of journal entries and interview transcripts were independently coded by two members of the investigative team to develop a coding scheme based on thematic analysis. Discrepancies were resolved via discussion among these two members, and in situations of greater uncertainty, with the rest of the research team. The coding scheme was used by the research coordinator and a research assistant to analyze the remaining data. All transcripts were coded and additional concepts arising during the later stages of analysis were added as new codes. A constant comparative process ${ }^{48,50}$ was used to compare 
and contrast findings to identify core concepts and processes related to the $\mathrm{KB}$ role and to identify organizational factors that facilitate or inhibit the process of research uptake.

\section{Partnerships for Health System Improvement (PHSI) Case Study (2009-2013)}

A case study, conducted in partnership with three Ontario health departments (i.e. cases), sought to evaluate the effectiveness of a 22-month KT intervention implemented by KBs. The research team worked with the decision-maker partners to tailor the KT intervention (e.g. largegroup training, one-on-one consultation, virtual support, consulting on policies and procedures, etc.) to the needs and goals of each health department. A small subset of health department staff (i.e. public health practitioners) were intensively involved with the KB (one-on-one mentoring to work through and apply the steps of EIDM to a practice-based question) and a larger subset (including the staff in the smaller subset) were involved in more introductory level EIDM workshops. The intervention provided in this case study included the same activities described above for the RCT. Data collection methods included quantitative (e.g. social network data, skills assessment and survey on knowledge/behaviour) and qualitative (e.g. interviews and knowledge broker journaling) components, at three time points (baseline, interim, follow-up). A total of 37 interviews were conducted, representing public health practitioners intensively involved with the $\mathrm{KB}(\mathrm{n}=14)$, managers $(\mathrm{n}=16)$, and senior management $(\mathrm{n}=7)$ with varied levels of experience, educational background, and length of time in position. Demographic data for participants in both studies is presented in Table 1. Qualitative data included participant reflections on: the successes and challenges of the intervention; how the KB had impacted themselves and their work; and their thoughts in general towards evidence-informed public health decision making and extent to which EIDM was occurring at the health department. 
Interviews were recorded and transcribed verbatim; fillers, ambient sounds, non-verbal communication, and all identifying information were omitted from final transcripts. The development and application of a coding scheme (Figure 1) for qualitative analysis of the interview transcripts followed the same process as described in the RCT. The research team met regularly to discuss the qualitative analysis, including resolving any discrepancies in coding and justifying the inclusion of new codes.

\section{Results}

The overall quantitative findings from the RCT illustrated that tailored messages led to a significant increase in the number of programs and policies supported by research evidence $(p<$ 0.01), while exposure to either the KB intervention or the healthevidence.org online registry, did not $(\mathrm{p}<0.45)$. The impact of the KB intervention was found to be moderated by organizational research culture (e.g. value placed on research evidence in decision making) ${ }^{45}$ However, in subgroup analysis, health departments exposed to the KB intervention that rated the organization low on a 7-point Likert scale on culture for EIDM at baseline, had a statistically significant increase in the number of programs and policies supported by research evidence at follow-up. These and additional quantitative results have been previously published. ${ }^{45}$

Quantitative results from the PHSI case study are currently in preparation for publication. Results illustrate that participants who worked closely with the KB showed a statistically significant change in knowledge and skill (2.8 points out of a possible 36 points ( $95 \%$ CI 2.0 to 3.6, $\mathrm{p}<0.001)$ ) from baseline. These participants also showed a statistically significant increase of $49 \%$ in EIDM behaviours from baseline $(\mathrm{p}<0.05)$. 
All of the participants interviewed, across the two studies, described their involvement with the KBs as a largely positive and helpful experience. The KB was called a "mentor", an “advocate", a "go-between", a "champion" and a "cheerleader". Several themes were identified from the qualitative analysis across both studies; these themes - enhancing capacity, effective KB attributes, and optimal positioning and ways of working - along with the challenges identified in implementing the KB role, are reported in greater detail below.

\section{Enhancing capacity}

The theme of enhancing capacity for EIDM emerged at two interrelated levels: individual and organizational capacity. Individual capacity refers to the knowledge, skills and behaviours, demonstrated by staff to search for, appraise and translate research evidence into practice. Organizational capacity refers to the structures, supports and overall culture at the health department, ultimately defining the environment in which EIDM is situated.

With respect to individual capacity, the majority of participants interviewed attributed their improved understanding of EIDM in general to the support and teaching of the KB in both the RCT and the PHSI case study. They appreciated that the KB was available to clarify any points of uncertainty throughout the process, which helped maintain momentum with their work and with their learning.

“...when we came across our stumbling blocks, then we were just able to consult with [the KB] and then move forward with a lot more confidence that we were doing the right thing and we could see that we weren't getting off track."- Public Health Practitioner 
All participants across the two studies felt that they were more skilled in searching, identifying, appraising, understanding, and applying research evidence in their daily work, to varied extents, after working with a KB. In both studies, the KB recommended relevant tools and resources and provided guidance on how to use and modify these resources. While participants identified the value of the resources themselves, it was the personal guidance on how to use and modify them that was most important. Many participants reported that they now had greater confidence in their ability to apply these skills and use these resources in their work moving forward.

Some participants in the two studies indicated that a key learning for them was understanding exactly what a KB was and how they could benefit from working with one. It was only after grasping the concept and purpose of a KB that participants were able to engage in the EIDM process with the KB. With respect to developing organizational capacity, this common understanding and role clarity was also required at the management level. In the PHSI case study, most participants identified the KB as a "champion" due to her role in liaising with management to ensure staff had protected time to meet with and learn from the KB. Participants across both studies identified ongoing management and senior management support as a critical component for supporting staff in using research evidence to inform policy and practice.

"I think the single most important influence...is the huge senior management and Board of Health support that we have...it makes all the difference"- Management

An approach to increase organizational capacity was observed in one health department in the PHSI case study where a decision was made to implement a "train the trainer" model to 
mentor health department staff to become internal KBs. In another health department, a committee was established with the objective of increasing awareness, knowledge and behaviours for EIDM across the organization. One of the committee's deliverables, with considerable input from the KB, was to develop a Policy \& Procedure manual to address EIDM in the organization, creating strategic goals and priorities moving forward. In all three health departments, the KB liaised with management to advocate for staff time and also guided management through the EIDM process themselves, with additional counseling on appropriate strategies to further support their staff and lead-by-example. These are all examples of strategies the health departments pursued in order to embed EIDM into planning and performance management activities across the organization.

\section{Knowledge Broker attributes}

Our analysis identified several key attributes or characteristics that were central to the KBs' ability to implement the interventions in both studies and impact capacity. These included tangible skills and past experience, as well as more intangible personal qualities (or "soft skills") that can be challenging to pinpoint and learn.

"[The KB] is very skilled in terms of appraising evidence and data extraction and that, but she's also very skilled at education and working with people at where they're at, and encouraging. So she was like a cheerleader, as well, so that was very helpful for me."- Management

The KBs in both studies were experienced public health practitioners with expertise in research methodology and the EIDM process. Participants commented that public health 
experience, coupled with the KBs' expertise in EIDM, gave the KBs credibility. Participants were able to develop respect for and trust in the KBs, which further supported their learning. An important attribute in both studies was the KBs' ability to pick up new knowledge quickly, in order to understand the practice-issue participants were addressing, and then be able to connect the practice with the research evidence. As the KB in the RCT described in a journal entry:

“...my role in [this] is trying to look at what's in the literature, what's available around [the issue] and practice around that issue... and then some very practical application of that to [the] context and setting. Sometimes there is literature on a particular topic but it may not transfer easily to [a particular] setting and context and so that's part of my role is trying to help find what's available and help with the contextualizing of that."'- KB

Participants also appreciated that the KBs could remain objective and provide unbiased direction.

“... it was more challenging for myself and the other [staff member] not to become emotionally invested in the answer. But [the KB] was much more objective. So she kept reminding us, "ok, remember, you're looking for the quote "truth" here..."- Public Health Practitioner

The majority of participants across both studies reported that the KBs were very "approachable", "responsive", "helpful" and "supportive", which enabled them to build a positive rapport and working relationship. The KBs were able to make participants feel comfortable, help them to deal with change, and mitigate anxiety inherent with the uncertainty of learning something new. Participants reflected on how the KBs were good "teachers", with clear 
communication skills, and the patience to walk them through and explain each step of the process, without simply "doing" the work for them.

"I don't think we would have gotten through the process if it hadn't been for [one of the KBs] mentoring. Just having somebody who is there that you can bounce questions - good, bad, ugly, stupid - off of, a face to ask those questions was really helpful. It took the stress levels down. "Public Health Practitioner

\section{Positioning and ways of working}

The final theme to emerge from this analysis relates to how the KB role was implemented or observed in each of the two studies. Participants reflected on the positioning of the KB in the health department, that is, whether the KB was an internal member of the organization or an external player. The KB in the RCT reflected that the $\mathrm{KB}$ being external to the organization may have contributed to her credibility and the trust that participants placed in her. Some participants in both studies appreciated that the KB was external to the organization in part because they felt safe bringing their questions to the KB and did not feel it would negatively impact their job if they admitted to not understanding something. The KB being external may have helped with the perception of the $\mathrm{KB}$ as an objective outsider, rather than someone to whom staff had to report and be accountable to in their position. While findings suggest that the KB being external to the organization was helpful, the critical factor here may be the neutrality of the KB. This could mean that an internal KB, as described below, may still be perceived as beneficial, providing the KB has no decision making or supervisory authority over those they are mentoring within the health department. Whether it is more important to be external as a $\mathrm{KB}$, or to not be in a position 
of authority over practitioners with whom a KB works, needs further exploration in future studies.

“... having her [the KB] as an external person, as somebody that you can go to and not feel... not threatened, but you know, you don't feel as... nervous about, you don't report to her."'- Public

\section{Health Practitioner}

The case may also be made for internal KBs with respect to enhancing organizational capacity. One of the KBs in the PHSI case study reflected on whether the work with staff would be sustainable because if the KB simply "parachutes" in to an organization, she takes all of her knowledge and support with her when she leaves. In addition, a KB in the PHSI case study thought that the trusting relationships she was able to develop with staff were in part because she had had a previous working experience with the health department and was familiar with many of the staff and with how the health department operated. She reflected that this may have been comparable to being an internal member of the organization, and may have therefore contributed to an increased level of comfort and understanding with health department staff.

Participants and the KBs also reflected on the ways of working, or the delivery of the KB role, across the two studies. Participants valued having a KB reliably available for in-person meetings, as well as one who was responsive and timely with virtual support (i.e. returning phone calls). It was important to participants that the KB had both the time to meet with them and the flexibility to accommodate their schedules. In both studies, the KBs reflected that it was more difficult to engage staff between visits, particularly at the beginning of the interventions before solid working relationships and an understanding of the KB role had been established. 


\section{Challenges to implementation}

We identified challenges, experienced at both the individual and organizational level, as a result of implementing the intervention in both studies. One challenge related to prioritization: at times, EIDM work was not deemed a priority and organizations therefore failed to acknowledge the need to allocate adequate time within the work day or to make changes to work load in order for participants to have sufficient time to spend with the KB and complete their EIDM work. Another challenge related to role clarity: in both studies, there were instances in which the KB had to resolve what the role entailed, resulting in periodic discussions about the role with the research team. Through these discussions, problem solving occurred to: help resolve what the KB should do in certain situations (e.g. how and when to advocate for participants' time and workload); instill confidence in the KB to be able to interact on all decision making levels, as required; and counsel the $\mathrm{KB}$ on working effectively with many different types of personalities.

\section{Discussion}

Although the $\mathrm{KB}$ role is no longer a new idea ${ }^{15}$, we continue to learn how KBs can work effectively. Brokering is thought to improve the quality and usefulness of evidence that is employed in decision making ${ }^{25}$ while promoting a decision making culture that values the use of evidence. $^{25,26,41}$ To date, there has been mainly anecdotal evidence that KBs produce positive effects on EIDM. Given that KBs represent an intensive and costly KT intervention, it is necessary to have rigorous evidence of effect before further implementation occurs. The studies reported here represent emerging data from rigorous research designs that support the anecdotal evidence that KBs hold promise as an effective strategy for EIDM. Here we provide an overview 
of insights from two studies implemented by our team from 2003-2013, along with supporting evidence from the literature on KBs. The following is a summary of contributions of the role, with broad recommendations for public health.

\section{Who is a Knowledge Broker?}

Using the best available evidence to create the infrastructure for evidence-based practice is more likely to result in success of the programs that are implemented. ${ }^{51}$ Although progress towards an evidence-informed culture has been reported ${ }^{52,53}$, the cultural shift required is demanding and requires accompanying knowledge management and communication strategies, along with monitoring, management and evaluation. ${ }^{54}$ A strategic approach that involves multiple strategies has been recommended. ${ }^{51}$ Our results illustrate that capacity building interventions are necessary but not sufficient. ${ }^{52}$ Although there is little consensus at this time on the exact nature of $\mathrm{KB}$ interventions ${ }^{55}$, it has been proposed that in order for effective knowledge translation to occur, expert facilitation must be employed. ${ }^{55}$

In the two studies described in this paper, the KBs were externally-positioned "mentors" who worked within health departments to enhance both staff and organizational capacity related to accessing, appraising, and applying research evidence to public health decision making. They were masters-prepared with 5-15 years' experience in public health. KB roles have been operationalized and implemented in different ways across organizations, and in some cases, KB positions have been filled with professionals newly graduated from undergraduate programs, meaning that practical work experience in the field in which they intend to work is limited, perhaps even lacking altogether. Additionally, there has been a tendency to hire KBs with communications and/or journalism backgrounds because the emphasis of the role has been on 
disseminating research results in user-friendly ways, rather than relationship building. Our results illustrate the importance of the KB having experience in the field in which they are doing KB work. Being viewed as similar to those with whom one works could be beneficial for the $\mathrm{KB}$, and this cultural compatibility has been reported as a key characteristic in a realist synthesis of change agency roles. ${ }^{37}$

The KB role leveraged both research background and credibility based in practical experience, as also described in the literature. ${ }^{14,56}$ Further to these external KBs, the idea of internal KBs arose. Specific staff were deemed to be well-positioned to become internal KBs because of their roles as consultants in a directorate that is mandated to provide planning and performance management support to staff in other directorates. These internal brokers currently focus, to varying degrees, on the full range of $\mathrm{KB}$ activities (described in this paper and in more detail elsewhere $\left.{ }^{45,46}\right)$; with additional training, it is expected that they will be a further asset in sustaining EIDM across the health department. ${ }^{57,58}$

\section{What are the attributes of a Knowledge Broker?}

The attributes of an effective KB identified in our studies are consistent with the literature to date, with emphasis on those attributes that enable successful personal interactions. ${ }^{58}$ This personal connection is important in collaborative work $^{56}$ and the success of a KB is at least partly personality-related. ${ }^{12,25,41}$ Attributes of successful KBs include personal qualities such as: approachability; comfort in dealing with people at multiple levels; ability to gain trust; and ability to adjust style to the person with whom they are working. ${ }^{14-20,59}$ Successful relationship building is central to successful knowledge brokering. $\mathrm{A} \mathrm{KB}$ also requires strong communication skills $^{15,17,60}$, knowledge of EIDM and the research process ${ }^{19}$, information management and 
synthesis $^{14,21,56,59}$, knowledge related to learning styles and teaching ${ }^{56}$, change management skills $^{14}$, ability to be non-judgmental and work in a respectful manner ${ }^{23}$, and a familiarity with the practice area. ${ }^{14,60}$ Personal characteristics are key to success ${ }^{37}$, and given the importance of interpersonal connection for the role, it is clear that attention must be paid not only to knowledge and skills of a broker, but these personal qualities that affect outcomes. ${ }^{37}$

Beyond translation and dissemination, a broker offers the added value of interaction to share their "know how" of implementation ${ }^{30,32,36}$, building on existing capacity to apply research evidence to local issues. A broker requires superior interpersonal ${ }^{27,61}$ and communication skills $^{20,25,27,34}$ to work effectively and collaboratively across contexts. These skills are needed for the $\mathrm{KB}$ to build and maintain rapport within the various communities of practice and networks in which they work. A combination of research knowledge and practical experience assists the broker in translating research evidence and establishing credibility, an essential quality in a broker. ${ }^{25,30,40,41}$ The KB should possess training and expertise from both decision makers' and researchers' domains. ${ }^{62}$

Qualifications for hiring a KB in public health should therefore include: 1) considerable practical experience working actively in public health; 2) good understanding of research concepts; and 3) personality or demeanor to facilitate constructive work with others, accompanied by a proven track record of doing so successfully. ${ }^{15,16,19,21}$

\section{When is a Knowledge Broker a good idea?}

Like knowledge brokering itself, a tailored approach to making the decision to implement a KB in an organization is needed. Promoting the use of research evidence in practice requires close attention to the interplay between individual and organization ${ }^{8}$ and interventions require 
commitment on multiple levels. ${ }^{52} \mathrm{~A}$ KB is well-placed to address these types of relationships in a customized manner. However, some considerations for the decision to implement a KB are: readiness (individual and organizational); current and desired EIDM work; momentum of work and ability to make time for new work, including learning; ability to champion the EIDM agenda with management; and the need to take a customized approach that attends to context, which is a key factor in knowledge exchange. ${ }^{63-67}$ A KB addresses much-needed individual (skills, knowledge, and behaviour) and organizational (management support, strategic goals and policies) capacity building ${ }^{14,56,59}$, which may be a way to position a KB to gain support and momentum in terms of addressing core competencies.

\section{How should a Knowledge Broker role be implemented?}

A KB's approach needs to be tailored to the individual and the situation, to address the context that forms a background for KBs' work $^{21}$ and should address multiple levels within the organization. One approach may be to implement an internal KB approach: identified staff members receive additional training and mentorship from an established KB; these staff can then provide support internally, and subsequently train additional (internal) KBs. Regardless of whether internal or external, the broker role requires well-developed problem solving, change facilitation, and change management skills. ${ }^{14}$ Accordingly, the KB then needs to be empowered by the organization to leverage factors that will facilitate EIDM, while at the same time address barriers. In the studies reported here, for example, the organizations' visible support of the KBs enabled them to better work with and build trusting relationships with staff, who were encouraged to participate. Partly due to the strength of these relationships, the KBs became aware of barriers identified by staff, such as work load and time constraints, and could advocate 
on their behalf. However, even with an understanding of type and degree of change, barriers, and facilitators, there is no clear, simple process. ${ }^{68}$

The organization should consider having a KB on-site for in-person support, even if only on a part-time basis, while recognizing that the potential for virtual support may be greater once relationships have developed. The organization needs to support a responsive and timely way of working, with the broker needing dedicated time to engage with staff. ${ }^{12}$ The role requires that an organization has given some careful thought to the approach and, in our experience, been actively involved in its implementation. Lomas (2007) suggested a KB role has both practical and philosophical dimensions to be considered: the philosophical, which makes KBs' activities part of a broader strategy ${ }^{16,59}$, including capacity development; and the practical, which supports KBs with tools, resources, and dedicated time. ${ }^{69}$

Brokers undertake a wide range of activities ${ }^{16,69}$, and there has been a call for role clarity $^{16,23}$ and to recognize and formalize the role. ${ }^{15}$ Senior leadership's role in taking a clear, strategic approach has been noted as a facilitator for a KB intervention in both studies described here. Additional organizational strategies that supported the KB intervention in some instances are: widespread organizational training projects with levels of training depending on requirements of different levels or types of staff; general orientation, with options for further training for senior management with respect to EIDM in particular; mentoring by an external $\mathrm{KB}$; and active promotion of the strategic priorities and organization's philosophy, so that the desired practices surrounding that philosophy are also clear.

The option to work jointly with private consultants as brokers may not be feasible for organizations due to costs. ${ }^{40}$ On a system wide level, it may be preferable to make a long-term goal of building KB capacity within a community and in the short-term, train a new group of 
specialist brokers focused on KT. Similar to mentoring individual decision makers, a tapering approach can be applied to establish and support communities which can become selfsustaining. ${ }^{32}$ Exposure to a $\mathrm{KB}$ offers potential to train new brokers later in the process ${ }^{27}$, and a broker's efforts can be directed at facilitating the evolution from decision maker to broker. ${ }^{22}$

Evidence-based practice has been proposed to operate best within an organizational context that supports practitioners ${ }^{53,68,70}$ and acknowledges the fears and anxieties that go along with change ${ }^{68}$ This involves setting in place the necessary structures ${ }^{68,71}$, workforce development $^{72}$, and the strong support of leadership. ${ }^{51,53,54,70}$ Implementation of these strategies for change requires ongoing, strategic efforts at the systems level ${ }^{52}$, with such a commitment being long-term and coming at a substantial cost. ${ }^{53}$

In terms of the time frame for a $\mathrm{KB}$ intervention, findings from the $\mathrm{RCT}$ indicate that a 12-month time frame is not sufficient to generate a measurable impact on programs and policies at the organizational level. ${ }^{23} \mathrm{~A}$ key lesson from the RCT was that the KB work was more complex than expected, taking more time to build trusting, collaborative relationships, and do the capacity building work needed. So while time to move staff along the EIDM continuum varied among health departments in the study, it was thought to be more likely that a multi-year intervention would be required to have an impact at the organizational level, requiring a corresponding long-term commitment (financial and resource) on the part of health departments. $^{45}$

Having increased the intervention time frame to 22 months for the PHSI case study, we can see that a broker does need that increased time to engage with staff to impact outcomes. Regularly scheduled face-to-face meetings (e.g. from twice a month in one health department, to twice a week in another) and ongoing virtual support and check-ins were imperative for the KB 
to build and maintain relationships with participants and to keep participants on track with their work. We are aware that once knowledge and skills are established, additional practice is also needed in order for staff to gain the confidence to lead the process internally, and that the learning and practice of EIDM takes time. The time required factors into the cost of implementing such an intervention, whether done by an internal or external broker.

\section{Who funds a position such as a Knowledge Broker?}

Cost is a potential barrier to the implementation of a KB; however, there are some organizations such as the National Collaborating Centers (NCC) that provide KB support whereby a broker employed by one of the six NCCs might facilitate the work of public health from an external agency. The internal broker is an idea that has not yet been implemented widely, but might be viewed as an organizational investment similar to investment in research and development. Although cost-benefit analyses were not conducted in either study, the authors were approached to provide ongoing service which could be an indication that there were perceived benefits that made KB services worth the investment. Another factor to consider is that through this approach, in some instances health departments have stopped implementing programs found to be ineffective through the work done with the $\mathrm{KB}$, resulting in the health department saving money. A cost-benefit analysis that directly addresses these factors would greatly assist in answering the question of whether a KB is a good investment for a particular organization at a specific time. To the authors' knowledge, no such study has been conducted to date.

\section{Why hire a Knowledge Broker?}


In this age of community engagement, with the emergence and focus of social media and the Internet as communication mainstays ${ }^{73}$, the linking function that is central to the $\mathrm{KB}$ role becomes incredibly important. A KB is well-positioned to address the multiple levels at which $\mathrm{KT}$ interventions work, and to facilitate an EIDM approach across those levels, addressing the widespread need for capacity development ${ }^{74}$ and possibly even turning those who may be resistant to EIDM into supporters. ${ }^{17}$ Additionally, a KB is well-positioned to train additional internal KBs and promote the sustainability of a KB intervention more broadly ${ }^{69}$ For public health and indeed all health care sectors KBs can help leverage existing resources, at whatever level they exist, to support a transition to EIDM. Given the benefits, it has been recommended that organizations consider KBs even when funds are low. ${ }^{17}$

Though a standard job description or widely-accepted KB qualifications do not currently exist, $\mathrm{KB}$ training programs are underway. ${ }^{56}$ There are also a growing number of venues for obtaining the skills and experience for EIDM in public health which are critical for KBs (e.g. National Collaborating Centre for Methods and Tools' online learning modules ${ }^{75-78}$; Public Health Agency of Canada's Skills Online program ${ }^{79}$; Canadian Centre for Evidence-Based Nursing's What is EIDM? Workshop ${ }^{80}$ ).

Measuring and interpreting the impact of KBs is not a clear task ${ }^{81}$; however, it is clear that effective brokers can increase self-reported knowledge and use of tools ${ }^{57}$, improve capacity, and increase the uptake of research evidence in contexts for which organizational support is perceived as low. ${ }^{14,59}$ Though a promising approach, feasibility and cost-effectiveness data are still needed. ${ }^{57}$ Knowledge brokering represents one possible KT strategy, though it has been proposed that multiple strategies are necessary. Continued study of brokering is needed to further inform implementation of the role and to address the concurrent challenge of evaluating KT 
interventions. ${ }^{82}$ Additional plans are underway to capture and share experience of the KB role, with the hope of advancing its application and future evaluation. 


\section{Acknowledgements}

The authors gratefully acknowledge the support of health department staff across Canada who have given their time and thoughtful feedback to contribute to this work.

\section{Funding}

The authors gratefully acknowledge funding from the Canadian Institutes of Health Research (RCT funding ref MOP-64201; trial registration ISRCTN35240937; PHSI case study - FRN 101867) and support, both financially and in-kind, from partner health departments on these studies.

\section{Competing interests}

Dr. Dobbins is the creator and Scientific Director of one of the current venues for knowledge brokers (Health Evidence), described in this paper. Otherwise, the authors declare that they have no competing interests.

\section{Ethical approval}

Ethics approvals for both studies described here were obtained from the Hamilton Health Sciences/McMaster Faculty of Health Sciences Research Ethics Board (HHS/FHS REB). Ethics approval was also received from the respective Research Ethics Boards of each of the

participating health departments. Informed consent was obtained from all participating staff prior to participation in these studies. 


\section{Reference List}

(1) Ciliska D, Dobbins M, Thomas H. Using systematic reviews in health services. In: Webb $\mathrm{C}$, Roe B, (eds). Reviewing research evidence for nursing practice: Systematic reviews. Malden, MA, USA: Wiley-Blackwell; 2007. p. 245-53.

(2) DiCenso A, Bayley L, Haynes RB. Accessing pre-appraised evidence: Fine-tuning the 5S model into a 6S model. Evid Based Nurs 2009;12(4):99-101.

(3) Ciliska D, Thomas H, Buffett C. An introduction to evidence-based public health and a compendium of critical appraisal tools for public health practice (Revised). Hamilton, Ontario, Canada: National Collaborating Centre for Methods and Tools; 2012.

(4) Baker AE, Brownson RC, Dodson EA. Use of evidence-based interventions in state health departments: A qualitative assessment of barriers and solutions. J Public Health Manag Pract 2010;16(6):E9-E15.

(5) Carroll DL, Greenwood R, Lynch KE, Sullivan JK, Ready CH, Fitzmaurice JB. Barriers and facilitators to the utilization of nursing research. Clin Nurse Spec 1997;11(5):207-12.

(6) Cochrane LJ, Olson CA, Murray S, Dupuis M, Tooman T, Hayes S. Gaps between knowing and doing: understanding and assessing the barriers to optimal health care. $J$ Contin Educ Health Prof 2007;27(2):94-102.

(7) Davis DA, Taylor-Vaisey A. Translating guidelines into practice: A systematic review of theoretic concepts, practical experience and research evidence in the adoption of clinical practice guidelines. Can Med Assoc J 1997;157(4):408-16.

(8) Squires JE, Moralejo D, LeFort SM. Exploring the role of organizational policies and procedures in promoting research utilization in registered nurses. Implement Sci 2007;2:17-27.

(9) Hutchinson AM, Johnston L. Bridging the divide: A survey of nurses' opinions regarding barriers to, and facilitators of, research utilization in the practice setting. J Clin Nurs 2004;13(3):304-15.

(10) Golden-Biddle K, Reay T, Petz S, Witt C, Casebeer A, Pablo A, et al. Toward a communicative perspective of collaborating in research: The case of the researcherdecision maker partnership. J Health Serv Res Policy 2003;8(Suppl 4):20-5.

(11) Ross S, Lavis J, Rodriguez C, Woodside J, Denis J-L. Partnership experiences: involving decision-makers in the research process. J Health Serv Res Policy 2003;8(Suppl 2):26-34. 
(12) Bowen S, Martens P. Demystifying knowledge translation: Learning from the community. J Health Serv Res Policy 2005;10(4):203-11.

(13) Canadian Foundation for Healthcare Improvement. Glossary of knowledge exchange terms. Available at: http://www.cfhi-

fcass.ca/PublicationsAndResources/ResourcesAndTools/GlossaryKnowledgeExchange.a spx;2012 [accessed 10.20.13]

(14) Gerrish K, McDonnell A, Nolan M, Guillaume L, Kirshbaum M, Tod A. The role of advanced practice nurses in knowledge brokering as a means of promoting evidencebased practice among clinical nurses. J Adv Nurs 2011;67(9):2004-14.

(15) Gagnon ML. Moving knowledge to action through dissemination and exchange. J Clin Epidemiol 2011;64(1):25-31.

(16) Lomas J. The in-between world of knowledge brokering. BMJ 2007;334:129-32.

(17) Lyons R, Warner G, Langille L, Phillips SJ. Piloting knowledge brokers to promote integrated stroke care in Atlantic Canada. Moving population and public health knowledge into action: A casebook of knowledge translation stories. Canadian Institutes of Health Research (CIHR) Institute for Population and Public Health; 2006.

(18) Straus SE, Tetroe JM, Graham ID. Knowledge translation is the use of knowledge in health care decision making. J Clin Epidemiol 2011;64(1):6-10.

(19) Urquhart R, Porter GA, Grunfeld E. Reflections on knowledge brokering within a multidisciplinary research team. J Contin Educ Health Prof 2011;31(4):283-90.

(20) Canadian Health Services Research Foundation. The theory and practice of knowledge brokering in Canada's health system. Canadian Health Services Research Foundation; 2003.

(21) Ward V, Smith S, House A, Hamer S. Exploring knowledge exchange: a useful framework for practice and policy. Soc Sci Med 2012;74(3):297-304.

(22) Thompson GN, Estabrooks CA, Degner LF. Clarifying the concepts in knowledge transfer: A literature review. J Adv Nurs 2006;53(6):691-701.

(23) Robeson P, Dobbins M, DeCorby K. Life as a knowledge broker in public health. JCHLA/JABSC 2008;29:79-82. 
(24) Waqa G, Mavoa H, Snowdon W, Moodie M, Schultz J, McCabe M, et al. Knowledge brokering between researchers and policymakers in Fiji to develop policies to reduce obesity: a process evaluation. Implement Sci 2013;8:74.

(25) Clark G, Kelly L. New directions for knowledge transfer and knowledge brokerage in Scotland: Office of Chief Researcher Knowledge Transfer Team briefing paper. Available at: http://www.scotland.gov.uk/Resource/Doc/69582/0018002.pdf; 2005 [accessed 10.20.2013]

(26) Hartwich F, von Oppen M. Knowledge brokers in agricultural research and extension. In: Graef F, Lawrence P, von Oppen M, (eds). Adapted Farming in West Africa: Issues, Potentials, and Perspectives. Stuttgart, Germany: Verlag Ulrich E. Grauer; 2000. p. 44553.

(27) Pyper C. Knowledge brokers as change agents. In: Lissauer R, Kendall L, (eds). New practitioners in the future health service: Exploring roles for practitioners in primary and intermediate care. London: Institute for Public Policy Research; 2002. p. 60-70.

(28) Hinloopen J. The market for knowledge brokers. Small Business Economics 2004;22:407-15.

(29) Loew R, Bleimann U, Walsh P. Knowledge broker network based on communication between humans. Campus-Wide Information Systems 2004;21(5):185-90.

(30) Zook MA. The knowledge brokers: Venture capitalists, tacit knowledge and regional development. International Journal of Urban and Regional Research 2004;28(3):621-41.

(31) Cillo P. Fostering market knowledge use in innovation: The role of internal brokers. European Management Journal 2005;23(5):404-12.

(32) Hargadon AB. Brokering knowledge: Linking learning and innovation. Research in Organizational behavior 2002;24:41-85.

(33) von Malmborg F. Networking for knowledge transfer: Towards an understnading of local authority roles in regional industrial ecosystem management. Business Strategy and the Environment 2004;13:334-46.

(34) Lyons R, Warner G. Demystifying knowledge translation for stroke research: A primer on theory and praxis. Atlantic Health Promotion Research Centre; 2005.

(35) Sverrisson A. Translation networks, knowledge brokers and novelty construction: Pragmatic environmentalism in Sweden. Acta Sociologica 2001;44(4):313-27. 
(36) Burnett S, Brookes-Rooney A, Keogh W. Brokering knowledge in organizational networks: The SPN approach. Knowledge and Process Management 2002;9(1):1-11.

(37) McCormack B, Rycroft-Malone J, DeCorby K, Hutchinson AM, Bucknall T, Kent B, et al. A realist review of interventions and strategies to promote evidence-informed healthcare: a focus on change agency. Implement Sci 2013;8(1):107.

(38) Pawlowski SD, Robey D. Bridging user organizations: knowledge brokering and the work of information technology. MIS Quarterly 2004;28(4):645-72.

(39) Sowe S, Stamelos I, Angelis L. Identifying knowledge brokers that yield software engineering knowledge in OSS projects. Information and Software Technology 2006;48(11):1025-33.

(40) van Kammen J., Jansen CW, Bonsel GJ, Kremer JA, Evers JL, Wladimiroff JW. Technology assessment and knowledge brokering: The case of assisted reproduction in The Netherlands. Int J Technol Assess Health Care 2006;22(3):302-6.

(41) Van Kammen J, De Savigny D, Sewankambo N. Using knowledge brokering to promote evidence-based policy-making: The need for support structures. Bull World Health Organ 2006;84(8):608-12.

(42) Conklin C, Hallsworth M, Hatziandreu E, Grant J. Briefing on linkage and exchange: Facilitating diffusion of innovation in health services. United Kingdom: RAND Corporation; 2008.

(43) Fitzgerald L, Ferlie E, Hawkins C. Innovation in healthcare: how does credible evidence influence professionals? Health \& Social Care in the Community 2003;11(3):219-28.

(44) Choi BCK, Pang T, Lin V, Puska P, Sherman G, Goddard M, et al. Can scientists and policy makers work together? J Epidemiol Community Health 2005;59(8):632-7.

(45) Dobbins M, Hanna SE, Ciliska D, Thomas H, Manske S, Cameron R, et al. A randomized controlled trial evaluating the impact of knowledge translation and exchange strategies. Implement Sci 2009;4(1):61.

(46) Dobbins M, Robeson P, Ciliska D, Hanna S, Cameron R, O'Mara L, et al. A description of a knowledge broker role implemented as part of a randomized controlled trial evaluating three knowledge translation strategies. Implement Sci 2009;4:23-4.

(47) Miles MB, Huberman AM. Qualitative data analysis: a sourcebook of new methods. 1984. 
(48) Patton Quinn M. Qualitative Research \& Evaluation Methods. 3rd ed. California: Sage Publications; 2002.

(49) Yin RK. Case study research: Design and methods. 5 ed. Thousand Oaks: Sage Publications; 1994.

(50) Hewitt-Taylor J. Use of constant comparative analysis in qualitative research. Nurs Stand 2001;15(42):39-42.

(51) Newhouse RP. Creating infrastructure supportive of evidence-based nursing practice: Leadership strategies. Worldviews on Evidence-Based Nursing 2007;4(1):21-9.

(52) Stetler CB, McQueen L, Demakis J, Mittman BS. An organizational framework and strategic implementation for system-level change to enhance research-based practice: QUERI series. Implement Sci 2008;3(1):30.

(53) Ward M, Mowat D. Creating an organizational culture for evidence-informed decision making. Healthcare Manage Forum 2012;25(3):146-50.

(54) Peirson L, Ciliska D, Dobbins M, Mowat D. Building capacity for evidence informed decision making in public health: a case study of organizational change. BMC Public Health 2012;12(1):137.

(55) Kitson AL. The need for systems change: Reflections on knowledge translation and organizational change. $J$ Adv Nurs 2008;65(1):217-28.

(56) Straus SE, Brouwers M, Johnson D, Lavis JN, Legare F, Majumdar SR, et al. Core competencies in the science and practice of knowledge translation: description of a Canadian strategic training initiative. Implement Sci 2011;6(1):127.

(57) Russell DJ, Rivard LM, Walter SD, Rosenbaum PL, Roxborough L, Cameron D, et al. Using knowledge brokers to facilitate the uptake of pediatric measurement tools into clinical practice: A before-after intervention study. Implement Sci 2010;5:92.

(58) Urquhart RL, Johnston GM, McVorran SM, Burge FI. Perspectives of an interdisciplinaryg research team to engage practice: lessons from a knowledge exchange trainee experience. Health Policy 2010;5(4):47-57.

(59) Ward V, Smith S, Carruthers S, Hamer S, House A. Knowledge brokering: Exploring the process of transferring knowledge into action. Medical Research Council University of Leeds; 2010. 
(60) Barwick MA, Boydell KM, Stasiulis E, Ferguson HB, Blase K, Fixsen D. Knowledge trasfer and implementation of evidence-based practices in children's mental health. Toronto, Ontario: Children's Mental Health Ontario; 2005.

(61) Greenhalgh T, Robert G, MacFarlane F, Bate P, Kyriakidou O. Diffusion of innovations in service organizations: Systematic review and recommendations. The Milbank Quarterly 2004;82(4):581-629.

(62) Canadian Health Services Research Foundation. Issues in linkage and exchange between researchers and decision makers. 4 total in series. Ottawa, Ontario, Canada: Canadian Health Services Research Foundation; 1999 Jan 5.

(63) Atun R, de Jongh T, Secci F, Ohiri K, Adeyi O. A systematic review of the evidence on integration of targeted health interventions into health systems. Health Policy Plan 2010;25(1):1-14.

(64) Aveling EL. Mediating between international knowledge and local knowledge: The critical role of local field officers in an HIV prevention intervention. Journal of Community and Applied Social Psychology 2011;21(2):95-110.

(65) Dopson S, FitzGerald L, Ferlie E. Understanding change and innovation in healthcare settings: Reconceptualizing the active role of context. Journal of Change Management 2008;8(3):213-31.

(66) Kitson A, Schultz TJ. Measuring the contxt of care in an Australian acute care hospital: a nurse survey. Implement Sci 2010;5(60).

(67) McCormack B, McCarthy G, Wright J, Slater P, Coffey A. Development and testing of the context assessment index. Worldviews on Evidence-Based Nursing 2009;6(1):27-35.

(68) Austin MJ. Strategies for transforming human service organizations into learning organizations: knowledge management and the transfer of learning. J Evid Based Soc Work 2008;5(3-4):569-96.

(69) Knight C, Lightowler C. Reflections of 'knowledge exchange professionals' in the social sciences: Emerging opportunities and challenges for university-based knowledge brokers. The Policy Press 2010;6(4):543-56.

(70) Cummings GG, Estabrooks CA, Midodzi WK, Wallin L, Hayduk L. Influence of organizational characteristics and context on research utilization. Nurs Res 2007;56(4):S24-S39. 
(71) Strickland RJ, O'Leary-Kelley C. Clinical nurse educators' perceptions of research utilization: barriers and facilitators to change.[Erratum appears in J Nurses Staff Dev. 2009 Sep-Oct;25(5):235]. Journal for Nurses in Staff Development 2009;25(4):164-71.

(72) Nicklin W, Stipich N. Enhancing skills for evidence-based healthcare leadership: The Executive Training for Research Application (EXTRA) program. Nurs Leadersh 2005;18(3):35-44.

(73) Shea BJ. A decade of knowledge translation research - what has changed? J Clin Epidemiol 2011;64(1):3-5.

(74) Advisory Committee on Population Health. Survey of public health capacity in Canada. 2002.

(75) National Collaborating Centre for Methods and Tools. Introduction to Evidence-Informed Decision Making. Hamilton, ON: National Collaborating Centre for Methods and Tools; 2010.

(76) National Collaborating Centre for Methods and Tools. Critical Appraisal of Intervention Studies. Hamilton, ON: National Collaborating Centre for Methods and Tools; 2010.

(77) National Collaborating Centre for Methods and Tools. Learning Module: Quantitative Research Designs 101: Addressing Practice-Based Issues in Public Health. Hamilton, ON: National Collaborating Centre for Methods and Tools; 2012.

(78) National Collaborating Centre for Methods and Tools. Searching for Research Evidence in Public Health. Hamilton, ON: National Collaborating Centre for Methods and Tools; 2013.

(79) Public Health Agency of Canada. Skills enhancement for public health online continuing education modules. Available at: http://www phac-aspc gc ca/sehsacss/training_modules-eng php; 2009 [accessed 10.20.2013]

(80) Canadian Centre for Evidence-Based Nursing (CCEBN). What is Evidence-Informed Decision Making (EIDM)? [workshop]. http://ccebn mcmaster ca/workshop html 2013

(81) Ward V, House A, Hamer S. Knowledge brokering: The missing link in the evidence to action chain? Evidence \& Policy 2009;5(3):267-79.

(82) Tetroe JM, Graham ID, Foy R, Robinson N, Eccles MP, Wensing M, et al. Health research funding agencies' support and promotion of knowledge translation: An international study. The Milbank Quarterly 2008;86(1):125-55. 
TABLE 1: Participant Characteristics

\begin{tabular}{|c|c|c|c|c|c|}
\hline \multirow{2}{*}{\multicolumn{2}{|c|}{ Demographic }} & \multirow[t]{2}{*}{$\begin{array}{l}\text { RCT } \\
(\mathrm{N}=\mathbf{1 0 8})^{*}\end{array}$} & \multicolumn{3}{|c|}{$\begin{array}{l}\text { Case study } \\
(\mathrm{N}=\mathbf{8 0 4})^{* *}\end{array}$} \\
\hline & & & Case A & Case B & Case $\mathrm{C}$ \\
\hline \multicolumn{2}{|c|}{$\begin{array}{l}\text { Number of staff who worked intensively } \\
\text { with KB }\end{array}$} & 30 & 51 & 12 & 17 \\
\hline \multicolumn{2}{|c|}{$\begin{array}{l}\text { Number of staff who attended } \\
\text { workshops } * * *\end{array}$} & - & 250 & 97 & 421 \\
\hline \multicolumn{2}{|c|}{ Number of staff involved in data collection } & 108 & 258 & 391 & 155 \\
\hline \multicolumn{2}{|l|}{$\rightarrow$ Female } & - & $89 \%$ & $89 \%$ & $82 \%$ \\
\hline \multirow[t]{4}{*}{$\rightarrow$ Education } & Diploma & - & $13 \%$ & $16 \%$ & $34 \%$ \\
\hline & Baccalaureate & - & $61 \%$ & $50 \%$ & $54 \%$ \\
\hline & Masters & - & $24 \%$ & $30 \%$ & $12 \%$ \\
\hline & Doctorate & - & $2 \%$ & $4 \%$ & $0 \%$ \\
\hline \multirow[t]{4}{*}{$\rightarrow$ Position } & $\begin{array}{l}\text { Public Health } \\
\text { Practitioner }\end{array}$ & $44 \%$ & $81 \%$ & $79 \%$ & $69 \%$ \\
\hline & Managers & $26 \%$ & $7 \%$ & $10 \%$ & $8 \%$ \\
\hline & $\begin{array}{l}\text { Senior } \\
\text { Management }\end{array}$ & $10 \%$ & $3 \%$ & $2 \%$ & $3 \%$ \\
\hline & Other & $20 \%$ & $9 \%$ & $9 \%$ & $20 \%$ \\
\hline \multicolumn{2}{|c|}{$\begin{array}{l}\rightarrow \text { Mean years of experience in public } \\
\text { health (SD) }\end{array}$} & $13(-)$ & $9(8)$ & $12(8)$ & $11(9)$ \\
\hline \multicolumn{2}{|c|}{$\rightarrow$ Mean years in current position } & 5 & - & - & - \\
\hline \multicolumn{2}{|c|}{$\rightarrow$ Number of participants interviewed } & 12 & 16 & 13 & 8 \\
\hline
\end{tabular}

* The RCT unit of analysis was at the health department level, with one staff member representing each health department. Of the 108 participating health departments, 30 worked intensively with the KB.

** The PHSI case study unit of analysis was both at the individual and organizational levels. Total individuals represent a mix of staff from each of the three participating health departments in the case study, as measured at follow-up.

*** Workshop training was delivered, in some instances (i.e. Case C), department-wide and was not restricted to study participants.

Abbreviations: RCT, randomized controlled trial; KB, knowledge broker; SD, standard deviation 